\title{
Orientaciones de meta y necesidades psicológicas básicas en el desarrollo de la Expresión Corporal en educación primaria: un estudio piloto Goal orientations and basic psychological needs in the development of Corporal Expression in primary education: a pilot study
}

\author{
M aría Papí M onzó, Salvador García Martínez, M iguel García Jaén, Alberto Ferriz Valero
} Universidad de Alicante (España)

\begin{abstract}
Resumen. El objetivo del presente trabajo fue anal izar el efecto de una aplicación didáctica de Expresión Corporal sobre las orientaciones de meta y las necesidades psicológicas básicas en el al umnado de primaria en las clases de Educación Física. 73 estudiantes de Educación Física (39 chicas y 34 chicos) con edades comprendidas entre los 9 y los 12 años (40PRI, n=24; 5PRI, $n=23 ; y$ 6PRI, $n=26$ ), pertenecientes a un colegio público de Educación Primaria de una línea participaron en este estudio. Sellevó a cabo unainter vención didáctica específica parael desar rollo de laExpresión Cor poral durante siete sesiones de 50 minutos, en la que se realizó apoyo a la autonomía, trabajo en grupo y creatividad del alumnado. Se evaluó el clima motivacional (POSQ) y las necesidades psicológicas básicas (BPNES) de todo el alumnado. Los resultados indican un aumento significativo del conjunto de la muestra en las variables dependientes autonomía $(p<.001)$ y competencia $(p=.021)$ tras la intervención. En chicas, todas las necesidades psicológicas básicas aumentaron significativamente su valor tras la intervención. No hubo efecto en el clima motivacional excepto en sexto curso de educación primaria. Por tanto, el desarrollo de la Expresión Corporal presenta una serie de beneficios para el bienestar psicológico en el alumnado, especialmente en las chicas.
\end{abstract}

Palabras clave. Tarea, ego, autonomía, competencia, relación con los demás, educación física, autodeterminación.

Summary. The objective of this work was to analyse the effect of a didactic application of corporal expression (Body Expression) on goal orientations and basic psychological needs in primary school students in Physical Education lessons. 73 Physical Education students ( 39 girls and 34 boys) aged between 9 and 12 years (4th PRI, $n=24 ; 5$ th $P R I, n=23$; and 6th PRI, $n=26$ ), belonging to a one-line State school of Education Primary participated in this study. A specific didactic intervention was carried out for the development of Body Expression during seven lessons of 50 minutes, in which support was given to autonomy, teamwork and creativity of the students. The motivational climate (POSQ) and basic psychological needs (BPNES) of all the students were evaluated. The results indicate a significant increase in the entire sample in the dependent variables: autonomy $(p<0.001)$ and competence $(p=0.021)$ after the intervention. In girls, all basic psychological needs increased significantly its value after the intervention. There was no effect on the motivational climate(atmosphere) except in the sixth grade of primary education. Therefore, the development of Body Expression presents a series of benefits for the psychological well-being in students, especially in girls.

Keywords. Homework, ego, autonomy, competence, relationship with others, physical education, self-determination.

\section{Introducción}

En las últimas décadas, han aparecido diversas teorías que hablan del favorecimiento del bienestar psicológico en el ámbito de la educación y especialmente en Educación Física. Dos de las más relevantes y conocidas son la Teoría de Metas de Logro (Nicholls, 1989) y la Teoría de la Autodeterminación (Ryan \& Deci, 2002), que han aparecido dentro del ámbito de la Educación Física como dos teorías cognitivas que explican el funcionamiento de los procesos motivacionales y el bien-

Fecha recepción: 09-09-20. Fecha de aceptación: 26-03-21

Salvador García M artínez

salvador.garcia@ua.es estar humano.

La Teoría de las metas de logro (TML) se basa en la búsqueda de habilidad y competencia por una persona (Nicholls, 1989). En este sentido, explica dos posturas claras con relación a la percepción del éxito: la orientación al ego y la orientación a la tarea. Las personas con unaorientación al ego consideran quetienen éxito cuando sus logros son buenos en comparación con los demás, es decir, se evalúa en comparación social y refuerzos basados en ser el mejor. Mientras tanto, las personas con una orientación a la tarea regulan su percepción de éxito en función del esfuerzo que empleen en conseguir la meta y los logros conseguidos. (Ames, 1992; N ewton et al., 2000). Estas diferentes percepciones influirán en la forma que los individuos consideran su «yo» en lo que 
respecta a su autoestima (Duda $\&$ Hall, 2001). Como indicaron Fox (1997) y Diener (1984), «la autoestima es un indicador significativo del bienestar mental y la cal idad de vida». Así, dado que, en un clima orientado a la tarea, la competencia se entiende de un modo autorreferenciado y los factores de los que depende dominio, mejora y trabajo duro- son relativamente más dominables por la persona, en este tipo de clima, es más probable que se alcancen mayores 0 más estables niveles de autoestima (Covington, 1992; Harter, 1999). Investigaciones anteriores en contextos educativos deportivos y físicos (Duda, 2001) han apoyado un vínculo entre las percepciones de un ambiente deportivo orientado a la tarea y una mayor autoestima, al mismo tiempo que la percepción de un clima que involucra a la orientación al ego ha probado ser un determinante negativo de la autoestima (Duda \& Kim, 1997)

Por su parte, la Teoría de la Autodeterminación (TAD) se ha convertido en uno de los campos de investigación más amplio y empleado en el contexto de la psicología, la cual hace hincapié en la motivación, la personalidad, el desarrollo y el bienestar humanos (Ryan et al., 2019). LaTAD está formada por seis mini-teorías actualmente. Una de ellas, la conocida como teoría de las N ecesidades Psicológicas Básicas (BPNS), habla de cómo la autonomía, la competencia y las relaciones sociales son consideradas las tres necesidades psicológicas básicas (NPB) para el bienestar psicológico (Ryan et al., 2019). La frustración de estas necesidades, de manera dura 0 permanente, asistiría directamente a varias formas de psicopatología (Vansteenkiste \& Ryan, 2013). La necesidad de competencia se refiere a ser efectivo 0 sentirse válido en diferentes actividades (Ryan \& Deci, 2002). La necesidad de relación con los demás implica la vivencia y sensación de hallarse conectado e integrado con los demás (Ryan \& Deci, 2000). En cuanto a la autonomía, sucede cuando el maestro delega en sus discentes la responsabilidad para actuar de forma perseverante en vistas de su propio aprendizaje 0 , como definen W illiams et al. (1997), se entendería como el nivel en el que los docentes reconocen la capacidad del alumnado y promueven su participación activa en las tareas de aprendizaje. Seconsidera, pues, un procedimiento metodológico activo por parte de los docentes que facilitaría la intervención del alumnado en su aprendizaje autodeterminado (Moreno-Murcia et al., 2012; Moreno-Murcia \& Sánchez-Latorre, 2016).

Con objeto de utilizar conjuntamente ambas teorías aplicadas a la educación, Giménez et al. (2013) afirman que un clima orientado a la tarea en contextos de Edu- cación Física puede ayudar a satisfacer las NPB al desarrollar formas de motivación más autodeterminadas (Barkoukis y Hagger, 2013) y de disfrute (Grastén et al., 2012).

En otro orden de ideas, dentro del área de Educación Física, la Expresión Corporal tiene un valor globalizador que permite trabajar y desarrollar los aspectosfísicos, intelectuales, afectivosy sociales, así como un carácter integrador (Ruano-Arriagada \& Barriopedro, 2006; Broquéet al., 2018; Lobo \&W insler, 2006), dado que no se reduce exclusivamente a aspectos perceptivo-motrices, sino que los une a los aspectos especificados anteriormente (Broqué et al., 2018; M ontávez, 2003; Rueda, 2004). Todo ello sucede a través de juegos y otras propuestas lúdicas, las cuales permiten adquirir óptimas actitudes e incluso al canzar una educación inclusiva ya sea realizándose individual o colectivamente (Cayón, 2017; M aset, 2012). Para ello, los docentes de la Educación Física deberán prevenir un amplio repertorio de actividades dinámicas que desarrollen el aprendizaje significativo en el alumnado (Gamonales, 2016). La Expresión Corporal es entendida como: «El lenguaje que se convierte en materia educativa y se utiliza para el desarrollo potencial de la capacidad expresiva del ser humano, fomentando el conocimiento personal, la comunicación interpersonal y la exteriorización de los sentimientos internos del individuo (comunicación introproyectiva), a través de gestos, posturas y movimientos expresivos» (Arteaga, 2003; López \& Sánchez, 2013).

Por otro lado, la Expresión Corporal se caracteriza por la existencia de modelos abiertos de respuesta y por el uso de métodos favorecedores de la creatividad y la imaginación como sistemas no directivos (López \& Sánchez, 2010; Pérez et al., 2012). La Expresión Corporal, mediante el análisis y aprendizaje de los usos de su objeto originario, el cuerpo (Martín, 2015; Schinca, 2000), así como a través de la expresión del movimiento y su técnica, buscasu razón como transmisor de emociones y permite encontrar un lenguaje corporal particular y per sonal (López \& Sánchez, 2013; M artín, 2015). A su vez, otros autores destacan que, si el lenguaje corporal es el objeto de estudio de la Expresión Corporal y el instrumento del que se vale es el cuerpo, el entendimiento y la toma de conciencia de ese cuerpo y sus facultades (posibilidades y límites) nos facilitara el desa rrollo de este (Camacho, 2002; Sánchez et al., 2011). No obstante, si se desea desarrollar el lenguaje corporal propio y singular, se deben eliminar normas y estereotipos para fomentar respuestas creativas (López \& 
Sánchez, 2012; Sánchez et al., 2011).

Por todo lo expuesto anteriormente, el objetivo del presente trabajo fue analizar los efectos de una aplica ción didáctica de Expresión Corporal en los valores de orientación a la tarea de la percepción del éxito y en las NPB en el alumnado de primariaen las clases de Educa ción Física. Considerando las características propias y las recomendaciones para el desarrollo de la Expresión Corporal descritas anteriormente, se formulan las siguientes hipótesis de partida:

Hipótesis 1 (H1). Un planteamiento didáctico espećfico, a través de la Expresión Corporal como eje de contenido, aumentará los valores de todas las N PB en el alumnado de sexto curso de educación primaria.

Hipótesis 2 (H2). Un planteamiento didáctico específico, a través de la Expresión Corporal como eje de contenido, aumentará los valores de orientación a la tarea en el alumnado de sexto curso de educación primaria.

\section{Metodología}

\section{Diseño}

La investigación fue llevada a cabo dentro del contexto de Educación Física durante el curso académico 2019-2020 (previamente a la pandemia COVID-19). El presente trabajo se plantea en base a un diseño cuasi experimental. Los grupos de clase fueron establecidos por el propio centro educativo, siguiendo criterios generales de agrupación, como la ratio de alumnos por grupo y sexo, entre otros. La intervención fue llevada a cabo por el mismo docente-investigador especialista en Educación Física en todos los grupos de tratamiento. Se llevó a cabo una intervención didáctica específica para el desarrollo de la Expresión Corporal durante siete sesiones de 50 minutos, en la que se favoreció el apoyo a la autonomía, el trabajo en grupo y la creatividad del alumnado. Los contenidos principales de esta intervención se establecieron a partir de conjuntos de contenidos de la siguiente manera:

$\emptyset$ Posturas del yoga.

$\varnothing$ Corrección de la postura.

$\emptyset$ La respiración

$\emptyset$ La flexibilidad

$\emptyset$ Expresión de sentimientos e ideas con el cuerpo.

$\emptyset$ Capacidad de moverse al ritmo de la música.

$\emptyset$ Representación teatral e imitación de animales y personajes.

$\emptyset$ El uso de la flexibilidad para desarrollar posturas no habituales. $\varnothing$ Capacidad de relajarse y controlar la respiración.

Capacidad para escuchar y cooperar con los compa ñeros.

$\varnothing$ Interés por la consecución de las posturas propuestas.

$\varnothing$ Esfuerzo y perseverancia por conseguir las actividades propuestas.

$\varnothing$ Cooperación, relación y coordinación entre compañeras-0s.

\section{Participantes}

La muestra de este estudio está formada por 73 estudiantes de Educación Física (39 chicas y 34 chicos) con edades comprendidas entre los 9 y los 12 años (4PPRI, n $=24 ; 50 \mathrm{PRI}, \mathrm{n}=23 ;$ y 6 oPRI, $n=26$ ), pertenecientes a un colegio público de Educación Primariade una línea educativa, situado en la localidad de San Vicente del Raspeig de la provincia de Alicante (España). Todas las familias fueron informadas con detalle sobre el presente estudio antes de la realización de las pruebas y la intervención. Los alumnos-as aportaron un consentimiento informado firmado por el padre, la madre o tutor-a autorizando su participación en el estudio y la publicación de los resultados deformaanónima, así como se solicitó la aprobación por el Comité de Ética de la Universidad de Alicante.

La totalidad del al umnado fue seleccionado en base a un criterio de muestreo por conglomerados no probabilístico, aprovechando el curso y el grupo en el que estaban matriculados. Los cuestionarios que mostraron patrones anómalos de respuesta compatibles con procesos de respuesta atíicicos no se incluyeron finalmente en el estudio (dos casos; 2,7\%). La detección de dichos patrones se realizó a través del análisis manual, uno por asignación de la misma respuesta a todos los ítems y otro por no asistir al menos al $80 \%$ de las sesiones (Sanchez-O liva et al. 2014).

\section{Instrumentos}

Percepción de éxito (POSQ)

Se utilizó la versión española (Martinez et al., 2006) del Cuestionario de percepción de éxito (Roberts \& Balague, 1991; Robertsetal., 1998) paramedir laorientación de meta de los participantes en la clase de Educa ción Física. El cuestionario, este compuesto de 12 ítems. Seis de ellos responden al factor «orientación al ego» (por ejemplo, «Cuando practico Educación Físicasiento que tengo éxito cuando mi actuación supera la del resto de alumnos-as») y los otros seis, al factor «orientación hacia la tarea» (por ejemplo, «Cuando practico Educa- 
ción Física siento que tengo éxito cuando rindo a mi mayor nivel de habilidad»). Las respuestas a dicho cuestionario se basan en una escala tipo Likert que oscilaba desde 0 (totalmente en desacuerdo) hasta 10 (totalmente de acuerdo).

N ecesidades psicológicas básicas (BPN ES).

Se empleó la versión española de la Escala de N ecesidades Psicológicas Básicas en el Ejercicio (BPNES) (Vlachopoulos\& M ichailidou, 2006) adaptadaal contexto de educación primaria (Moreno et al., 2008). Este cuestionario contaba 12 ítems juntados en tres factores (cuatro ítems por factor) que midieron la autonomía (por ejemplo, «tengo la oportunidad de elegir cómo realizar los ejercicios»), la competencia (por ejemplo, «Siento que he tenido una gran progresión con respecto al objetivo final que me he propuesto" ) y la capacidad de relación (por ejemplo, "Me relaciono de forma muy amistosa con el resto de las compañeras-os"). Se trata de un cuestionario de escala tipo Likert que oscilaba entre 0 (totalmente en desacuerdo) y 5 (totalmente de acuerdo)

\section{Procedimiento}

En primer lugar, se aplicó una unidad didáctica de trabajo de Expresión Corporal, basada en estilos de enseñanza que favorecieran la autonomía y la creatividad del alumnado. Además de estos, se utilizaron otros estilos de enseñanza como la asignación de tareas, el traba jo en pequeños grupos, el descubrimiento guiado, el aprendizaje cooperativo y la resolución de problemas (Mosston, 1978; Delgado-N oguera, 1991).

Antes de llevarse a cabo, esta unidad de trabajo fue revisada y aprobada por cada uno de los investigadores, por otro maestro especialista en educación física del centro y por el equipo directivo. El tutor o tutora de cada curso administró los cuestionarios al al umnado impresos en papel, animando a que respondieran con la mayor sinceridad posible y recal cando que sus respuestas serían totalmente anónimas y que no influirían en sus calificaciones, ya que los datos serían gestionados de manera confidencial. Dispusieron de todo el tiempo necesario para cumplimentar adecuadamente los dos cuestionarios. El tiempo aproximado que emplearon fue de 15 minutos.

La intervención duró tres semanas y media, esto es, siete sesiones de $50^{\prime}$ en cada curso. Fue llevada a cabo por la docente que creó la unidad de trabajo haciendo siempre hincapié en la importancia del uso de la imaginación, de la creación autónoma y la compartición de ideas para un buen trabajo cooperativo. En el Anexo I se explica con más detalle el desarrollo específico de cada sesión.

Al acabar cada sesión, se realizó una asamblea conjunta donde se preguntaba al alumnado acerca de la sesión. Los temas que se debatieron estaban relacionados con sus sentimientos, sus emociones, sus percepciones, nuevos conocimientos, puntos a favor y en contra del planteamiento... con el fin de realizar una evaluación cual itativa general de la intervención llevada a cabo.

Finalmente, tras acabar la intervención didáctica, se rellenaron nuevamente los cuestionarios detallados en la sección Instrumentos. Una vez más, fue el tutor-a de cada curso quien realizó la distribución y recordatorio de su funcionamiento, la importancia de la sinceridad en ellos y la falta de repercusiones en su expediente y personal al rellenarlos, dado su anonimato. La recogida de los cuestionarios en ambos casos fue individual, verificando que ningún ítem se quedara sin contestar.

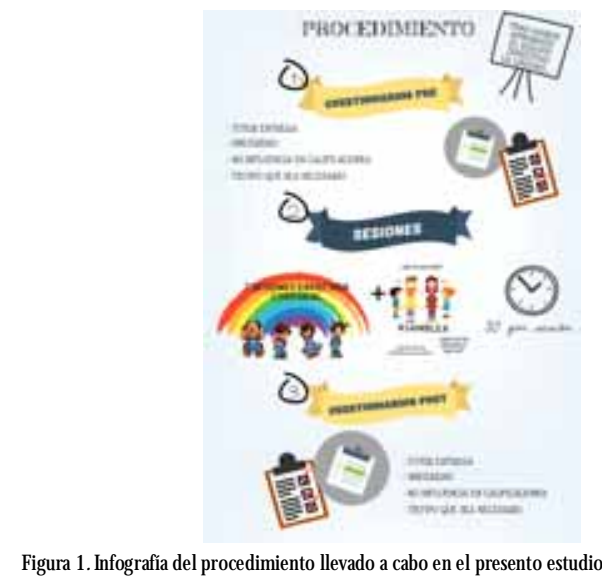

\section{Análisis estadístico}

Todas las variables continuas del conjunto de datos fueron sometidas a una prueba de normalidad (ShapiroW ilk). Los datos fueron sometidos a un análisis estadístico univariante para muestras no paramétricas, concretamente se utilizó la pruebaW ilcoxon para observar las diferencias intragrupo (pre vs post). El nivel de significación fue establecido en $p<.05$ en todos los ca sos. Se hizo uso del programa estadístico Statistics Product and Service Solutions (IBM $₫$ SPSS $®$ Statistics Versión 24.0.0.0) y Microsoft Excel ${ }^{\circledR}$ en su versión 2016.

\section{Resultados}

Tanto la variable dependiente autonomía $(p<.001)$ como la competencia $(p=.021)$ aumentaron significativamente tras la intervención. Se observa una 
tendencia a la significación en la variable relación con los demás ( $p=.087)$. En el resto de las variables no mostra ron cambios estadísticamente significativos.

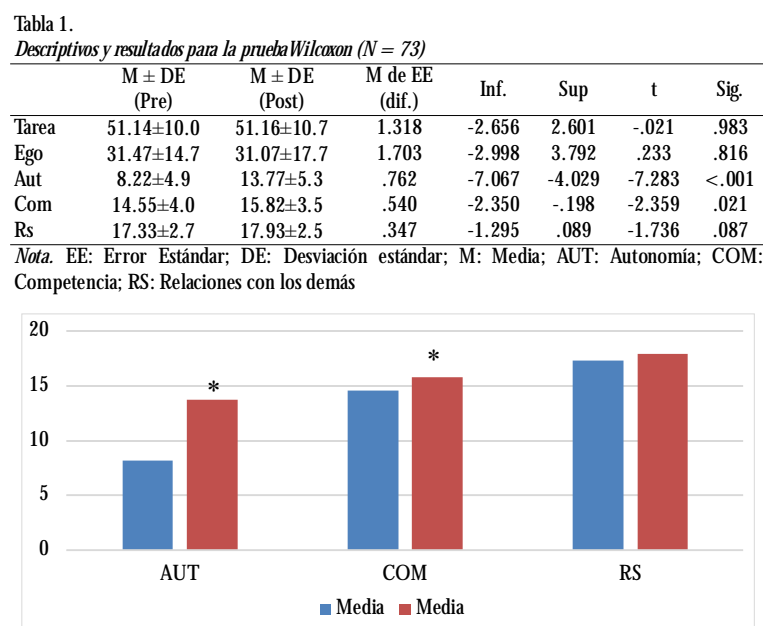

Figura 2. Gráfico de barras apiladas representativo de la media de los val ores de las necesidades psicológicas básicas, antes y después de la intervención para el conjunto total de estudiantes. (* psicologicas
$\mathrm{p}<0.03)$.

Analizando los resultados atendiendo a la variable independiente género (Tabla 2), se observa un aumento significativo traslaintervención en autonomía $(p<.001)$, competencia $(p<.001)$ y relaciones con los demás $(p=.031)$ sexo femenino. Por su parte, solamente la autonomía ( $p$ $=.018$ ) aumentó en el sexo masculino.

Tabla 2.

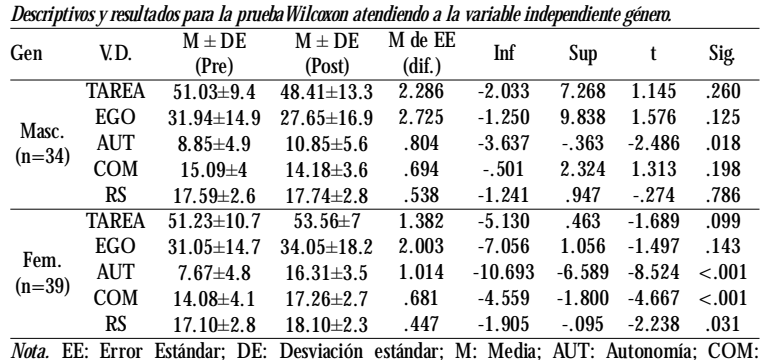
Nota. EE: Error Estándar; DE: Desviación estándar; M: Media; AUT: Autonon
Competencia; RS: Relaciones con los demás; Masc: Masculino; Fem: Femenino

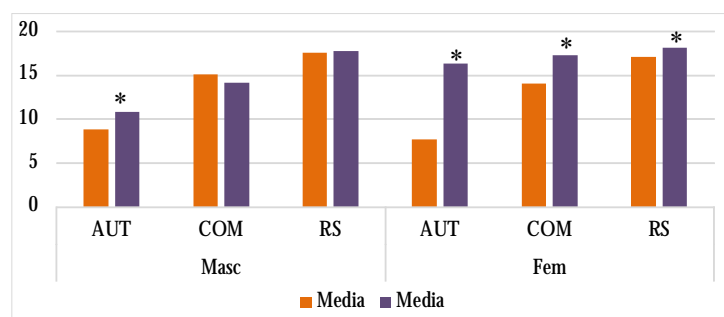

Figura 3. Gráfico de barras apiladas representativo de la media de los valores de las necesidades psicológicas básicas, antes y después de la intervención segmentado según la variable independiente género $(* 0<0.03)$.

Analizando los resultados atendiendo a la variable independiente nivel educativo (Tabla 3), se observa un descenso significativo tras la intervención en las variables dependientes orientación a la tarea en cuarto curso de educación primaria ( $p=.010$ ) y un aumento en sexto curso de educación primaria $(p=.019)$, en competen- cia en sexto curso de educación primaria $(p=.019)$ y en autonomía en cuerto curso de educación primaria ( $p$ $=.009)$, quinto curso de educación primaria $(p<.001)$ y sexto curso de educación primaria $(p<.001)$.

\begin{tabular}{|c|c|c|c|c|c|c|c|c|}
\hline $\begin{array}{l}\text { Nivel } \\
\text { académico }\end{array}$ & V.D. & $\begin{array}{c}\mathrm{M} \pm \mathrm{DE} \\
(\mathrm{Pre})\end{array}$ & $\begin{array}{c}\mathrm{M} \pm \mathrm{DE} \\
\text { (Post) }\end{array}$ & $\begin{array}{c}\text { M de EE } \\
\text { (dif.) }\end{array}$ & Inf & Sup & $\mathrm{t}$ & Sig. \\
\hline \multirow{5}{*}{$\begin{array}{c}\text { 40PRI } \\
(n=24)\end{array}$} & TAREA & $54.54 \pm 6.4$ & $47.08 \pm 14.8$ & 2.642 & 1.992 & 12.924 & 2.823 & .010 \\
\hline & EGO & $33.92 \pm 14.8$ & $34.92 \pm 16.0$ & 3.690 & -8.633 & 6.633 & -.271 & .789 \\
\hline & AUT & $8.00 \pm 5.0$ & $12.38 \pm 7.0$ & 1.527 & -7.534 & -1.216 & -2.865 & .009 \\
\hline & COMP & $16.38 \pm 3.6$ & $16.21 \pm 4.0$ & .898 & -1.691 & 2.025 & .186 & .854 \\
\hline & RS & $17.25 \pm 2.8$ & $17.75 \pm 3.0$ & .757 & -2.065 & 1.065 & -.661 & .515 \\
\hline \multirow{5}{*}{$\begin{array}{l}\text { 50PRI } \\
(n=23)\end{array}$} & TAREA & $51.30 \pm 8.2$ & $54.52 \pm 4.6$ & 1.726 & -6.797 & .363 & -1.864 & .076 \\
\hline & EGO & $25.04 \pm 12.9$ & $21.74 \pm 14.9$ & 3.076 & -3.074 & 9.683 & 1.074 & .294 \\
\hline & AUT & $9.91 \pm 4.5$ & $13.39 \pm 3.2$ & .792 & -5.122 & -1.835 & -4.389 & $<.001$ \\
\hline & $\mathrm{COM}$ & $14.30 \pm 3.6$ & $15.26 \pm 3.0$ & .865 & -2.751 & .838 & -1.105 & .281 \\
\hline & RS & $18.43 \pm 1.8$ & $19.00 \pm 1.2$ & .435 & -1.467 & .336 & -1.300 & .207 \\
\hline \multirow{5}{*}{$\begin{array}{c}\text { 60PRI } \\
(n=26)\end{array}$} & TAREA & $47.85 \pm 13.0$ & $51.96 \pm 9.0$ & 1.646 & -7.505 & -.726 & -2.501 & .019 \\
\hline & EGO & $34.88 \pm 14.7$ & $35.77 \pm 19.0$ & 2.022 & -5.050 & 3.281 & -.437 & .666 \\
\hline & AUT & $6.92 \pm 4.6$ & $15.38 \pm 4.4$ & 1.296 & -11.132 & -5.792 & -6.527 & $<.001$ \\
\hline & $\mathrm{COM}$ & $13.08 \pm 4.4$ & $15.96 \pm 3.4$ & .952 & -4.845 & -.925 & -3.031 & .006 \\
\hline & RS & $16.42 \pm 2.9$ & $17.15 \pm 2.6$ & .583 & -1.932 & .470 & -1.253 & .222 \\
\hline
\end{tabular}

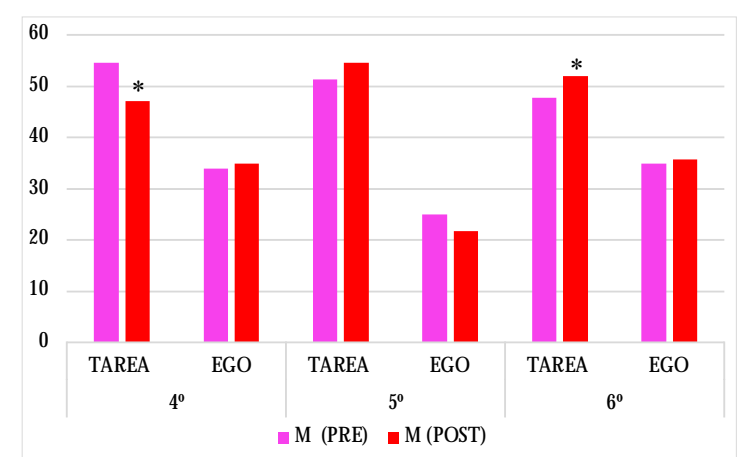

Figura 4. Gráfico de barras apiladas representativo de la media de los valores de las variables Tarea y Ego, antes y después de la intervención segmentado según la variable independiente nivel educativo $(* p<0.03)$.

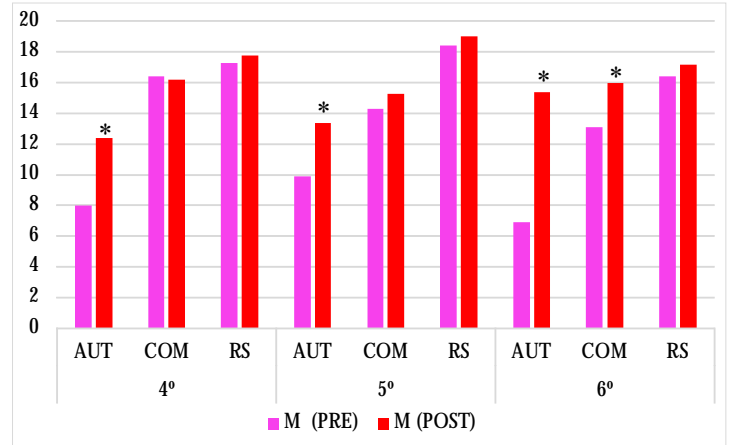

Figura 5. Gráfico de barras apiladas representativo de la media de los val ores de las necesidades psicológicas básicas, antes y después de la intervención segmentado según la variable independiente nivel educativo $(* p<0.01)$

\section{Discusión}

El principal objetivo de este estudio fue evaluar los efectos en los valores de orientación a la tarea de la percepción del éxito y las NPB de un programa de intervención utilizando una unidad didáctica basada en contenidos de Expresión Corporal, dirigida a alumnado de Educación Física de primaria (9-12 años).

Se partió de varias hipótesis iniciales que afirmaban que la intervención desarrollada generaría una mejora de todas las N PB (autonomía, competencia y relación). 
Los resultados obtenidos en la presente investigación aceptan parcialmente esta hipótesis ya que, como se observa en los resultados (Tabla 1), tanto la autonomía como la competencia han aumentado significativamente $(p<.05) y$, en la variable relación con los demás se muestra una tendencia a la significación ( $p=.087$ ) para todo el conjunto de los estudiantes. La autonomía es la varia ble que más cambio ha recibido después de la intervención. Este cambio podría deberse a que las actividades llevadas a cabo en la U nidad Didáctica, las cuales permitían al alumnado decidir y personal izar la ejecución, por lo que el sentimiento de cada estudiante podría ser demayor responsabilidad en su comportamiento y como consecuencia, un aumento de esta variable (Reeve, 2006). De la misma forma que la variable autonomía, la competencia del alumnado se ha visto aumentada debido al planteamiento de diferentes niveles de consecución de resultados de la técnica, lo cual hizo que estos se esforzaran por alcanzar metas progresivas y graduadas y así, se sintieran más competentes (Jang et al., 2010). Por último, a pesar de no observar cambio estadísticamente significativo, la creación de un clima relajado, cooperativo y de respeto, proporcionado por ladidácticade laExpresión Corporal (Vallés, 2000) pudo conllevar a una mayor integración de los discentes (Maset, 2012), aumentando así las relaciones sociales y el bienestar del alumnado (Tessier et al., 2010).

Por otro lado, se destaca una diferencia en el aumento de las NPB entre chicas y chicos, en favor de las chicas. A pesar de queW hitehead $\&$ Biddle (2008) afirman que el bienestar psicológico en las clases de Educa ción Física es mayor en los chicos que en las chicas, los resultados del presente trabajo no pueden apoyar esta afirmación ya que las chicas han aumentado de forma significativa todas las NPB mientras que los chicos sólo han aumentado la autonomía. Puede que estos resulta dos se deban a que el bienestar puede verse influenciado en función del tipo de metodología aplicada 0 área de contenido involucrada(M itchell et al. , 2013; Lee, 2002; SaM pol et al., 2005). Esto es de una importancia vital en el eje temático de Expresión Corporal, que en muchas ocasiones es poco aceptado por el alumnado masculino (Couturier et al., 2007; Gabbei, 2004; O'N eill et al., 2011). Debido a razones socioculturales, las niñas son las que principalmente se atribuyen a este tipo de área de contenido (Barness, 2001; Ruiz et al., 2010), por lo que se sienten más competentes, a diferencia de los chicos, que sienten que no es una disciplina de interés para ellos (Murillo et al., 2014). En línea con esto, existen ciertos prejuicios que, habitualmente, generan una actitud de desinterés, rechazo y falta de entusiasmo entre los alumnos masculinos hacia el desarrollo de la Expresión Corporal (Graupera \& M Mata 2004; O'N eill et al. 2011; Riemer $\&$ Viso, 2003). Esto se ve reflejado en las declaraciones, que ellos hacían en las asambleas, al gunas de ellas estereotipadas (p.e. : «... a mí no me gusta esto porque es como bailar y eso es de chicas", "estas cosas las hace mi madre y yo quiero ser como mi padre», «ellas tienen ventaja porque son chicas, se les da mejor»). No obstante, es de destacar que, a medida que avanzaba el número de sesiones, la investigadora que implementó la unidad de trabajo percibió cómo los chicos se sentían más cómodos, participativos y activos, aunque este dato es meramente subjetivo. Además, se ha de considerar que, aunque las diferencias entre chicos y chicas parezca tener sentido y pueda quedar abalado por la literatura presentada, también es cierto que la mayor o menor acepta ción de los contenidos presentados en función del género también puede depender de la selección de los contenidos a trabajar en la unidad didáctica. Así pues, podría ser que un planteamiento didáctico más extenso, 0 seleccionando diferentes contenidos de Expresión Corporal, pudiera aumentar en mayor medida el sentimiento de competencia en los chicos, tal y como observaron Sevil et al., (2016) en alumnado de primer ciclo de secundaria.

En otro orden de ideas, se planteó una segundahipótesis, la cual postulaba que nuestra intervención basada en EC puede aumentar la orientación a la tarea en la percepción del éxito del alumnado. Los resultados obtenidos en la investigación rechazan parcialmente esta hipótesis, ya que de acuerdo con los resultados mostrados en la Tabla 1, la orientación a la tarea no se ha visto aumentada de forma significativa de forma homogénea. M ientras que ha llegado a disminuir en cuarto curso de educación primaria, en 60 curso han aumentado significativamente dichos valores. En este sentido, pese a que Braithwaite et al., (2011) indican que los rasgos propios de la Expresión Corporal (variedad en las actividades, desafío personal, posibilidad de adquisición de responsabilidades y de toma de decisiones por parte de los discentes, tolerancia de diversos formatos de agrupación, etc.) son efectivos para la generación de un clima orientado a las tarea, los resultados obtenidos sólo apoyarían parcialmente que esto sea de esta forma y podrían indicar por tanto que serían necesarias metodologías más específicas, como la llamada metodología «TARGET» (Se ha descubierto que las estrategias basadas en estructuras de tareas, autoridad, reconocimiento, agrupación, evaluación y tiempo (TARGET) 
de las situaciones de logro influyen en el clima motivacional del aula), (M organ et al, 2005). Desde este punto de vista, esto apoyaría las conclusiones llevadas a cabo por Sevil et al. (2016), los cuales obtuvieron un aumento de la orientación a la tarea a través de una intervención de Expresión Corporal en alumnado de secundaria mediante el uso de esta metodologíaTARGET. El diseño de unaintervención con estetipo demetodologías en Educación Física ayudaría a aumentar la orientación a la tarea, del mismo modo que ha ocurrido en numerosos estudios que las emplean (Almolda Tomás et al., 2014; Barkoukis et al. , 2008; Cecchini et al., 2014; Cuevas et al. , 2012; M organ y Kingston, 2008) por lo que se anima a incluirlas de cara a futuras líneas de investigación en el desarrollo de la Expresión Corporal, ya que existe actualmente escasa literatura al respecto y no se pueden hacer comparaciones rigurosas con el fin de extraer conclusiones más sólidas.

Por último, cabe señalar que este estudio presenta como limitaciones la corta duración de la inter vención, así como la falta de grupo control. Por una parte, es probable que una intervención más larga hubiera favorecido un mayor aumento de los valores en relación con la orientación a la tarea de la percepción del éxito y a las NPB, por lo que esta hipótesis habrá que probarla en futuros estudios. Por otra parte, la falta de inclusión de un grupo control en el diseño experimental hace que los resultados del presente estudio no puedan ser generalizados directamente, y requieran por tanto de futuras investigaciones que corroboren estos resultados. Finalmente, sería interesante añadir, como futuras líneas de investigación, más variables de estudio como la motivación de los estudiantes o la percepción de la autoestima, así como aumentar la duración de la unidad de trabajo y del tiempo de intervención para indagar más profundamente en tales variables dentro de la Expresión Corporal.

\section{Conclusiones}

El desarrollo de la Expresión Corporal en esta aplicación didáctica presenta una serie de beneficios y ca racterísticas propias que parecen ser de gran ayuda a la hora de mejorar las N PB del alumnado, especialmente en las chicas. La Expresión Corporal es una disciplina que se ha visto envuelta en un conjunto de prejuicios y normas sociales estereotipadas, lo cual puede explicar estas diferencias encontradas entre sexos. Por último, se ha demostrado que, a pesar de sus características integradoras y globalizadoras, la Expresión Corporal necesita unirse a otras variables para conseguir aumentar la orientación a la tarea, como podrían ser metodologías más específicas que las empleadas en la presente intervención.

\section{Referencias}

Aijón, M. V. (2012). La expresión corporal, un camino tortuoso. EmásF: revista digital de educación física, (14), 9-26.

Almolda-Tomás, F. J., Servil-Serrano, J., Julián-Clemente, J., A barca-Sos, A., \& García González, L. (2014). Application of teaching strategies for Improving student' situactional motivation in physical education. Electronic Journal of Research in Educational Psychology, 12(2), 391-418.

Ames, C. (1992). Classrooms: goals, structures, and student motivation. Journal of Educational Psychology, 84, 261-271. https:/ / doi.org/ 10.1037/ 00220663.84 .3 .261

Arteaga, M. (2003). Fundamentos de la Expresión Corporal. Ámbito pedagógico. Grupo Editorial Universitario. Granada.

Barkoukis, V., \& Hagger, M. S. (2013). The transcontextual model: perceived learning and performance motivational climates as analogues of perceived autonomy support. European Journal of Psychology of Education, 28(2), 353-372. DO I:10.1007/ s10212-012-0118-5

Barkoukis, V., T sorbatzoudis, H., \& Grouios, G. (2008). European Physical Education.

Bowler, M. (2009, September). The influence of the TARGET motivational climate structures on pupil physical activity levelsduring year 9 athletics lessons. In British educational research association annual conference, university of Manchester, 2- 5 September (pp. 1-20).

Braithwaite, R., Spray, C. M., \&Warburton, V. E. (2011). Motivational climate interventions in physical education: A meta-analysis. Psychology of Sport and Exercise, 12(6), 628-638. https:/ / doi. org/ 10.1016/ j.psychsport.2011.06.005

Broqué, Y.Y. C., Castro, A. D. M., \& Vázquez, L. L. L. (2018). La expresión corporal como alternativa del Deporte paraTodos. Selección de ejercicios. Lecturas: Educación Física y Deportes, 23(239), 77-86.

Cabbei, R. (2004). A chieving balance: Secondary physical education gender-grouping options. J ournal of Physical Education, Recreation \& D ance, 75(3), 33-39.

Camacho, M. D. M. O. (2002). Expresión Corporal: Una 
propuesta didáctica para el profesorado de Educación Física. Grupo Editorial Universitario

Cayón, A. C. (2017). Prácticas innovadoras e inclusivas para la enseñanza de la expresión corporal en educa ción física. In Prácticas innovadoras inclusivas: retos y oportunidades (pp. 1251-1258). Servicio de Publica ciones.

Cecchini, J. A., Fernandez-Rio, J., Mendez-Gimenez, A., Cecchini, C., \& Martins, L. (2014). Epstein's TARGET framework and motivational climate in sport: effects of a field-based, long-term intervention program. International Journal of Sports Science\& Coaching, 9(6), 1325-1340. https: / / doi.org/ 10.1260/ 1747-9541.9.6.1325

Couturier, L. E., Chepko, S. \& Coughlin, M. A. (2007). $W$ hose gym is it? Gendered perspectives on middle and secondary school physical education. Physical Educator, 64(3), 152.

Covington, M. V. (1992). Making the grade: A self-worth perspective on motivation and school reform. Cambridge University Press.

Cuevas, R., Contreras, 0., \& García-Calvo, T. (2012). Effects of an experimental program to improve the motivation in physical education of Spanish students. Procedia-Social and Behavioral Sciences, 47, 734-738. https:/ / doi. org/ 10.1016/ j. sbspro. 2012.06.726

Deci, E. L., \& Ryan, R. M. (2000). The «W hat» and «W hy» of goal pursuits: Human needs and the selfdetermination of behavior. Psychological Inquiry, 11, 227-268. doi:10.1207/ S15327965PLI1104_01

Deci, E. L., \& Ryan, R. M. (2002). O verview of selfdetermination theory: An organismic dialectical perspective. Handbook of self-determination research, 333.

Delgado, M. A. (1991). Los estilos de enseñanza en la Educación Física. Propuesta para una Reforma de la Enseñanza. Granada: ICE de la U niversidad de Granada.

Diener, E. (1984) Subjective well-being. Psychological Bull etin, 95, 542-574.

Duda, J.L. (2001). A chievement goal research in sport: Pushing the boundaries and clarifying some misunderstandings. In G.C. Roberts (Ed.), Advances in motivation in sport and exercise (pp. 129-182). Champaign, IL: Human Kinetics.

Duda, J. L. , Balaguer, I. M oreno,Y., \& Crespo, M. (2001). The relationship of the motivational climate and goal orientations to burnout among junior elite tennis players. AAASP 2001 Conference Proceedings (p.70). Denton, TX: Ronjon Publishing.
Duda, J.L., \& Hall, H.K. (2001). A chievement goal theory in sport: Recent extensions and future directions. En R.N. Singer, H.A. Hausenblas, C.M. $\&$ Janelle (Eds.), Handbook of Research in Sport Psychology (2ª ed.; pp. 417-434). Nueva York: John $W$ iley and Sons, Inc.

Duda, J. L., \& Kim, M. S. (1997). Perceptions of the motivational climate, psychological characteristics, and attitudes toward eating among young female gymnasts. Journal of Sports and Exercise Psychology, 19 (Suppl.), S48.

Fox, K.R. (1997). The physical self and processes in selfesteem development. En K.R. Fox (Ed. ), Thephysical self (pp. 111-139). Champaign, IL: Human Kinetics.

Gamonales-Puerto, J. M. (2016). La Educación Física como herramienta de inclusión. Publicaciones Didácticas, 70(1), 26-33.

Gråstén, A. , Jaakkola, T., Liukkonen, J., Watt, A., \&YliPiipari, S. (2012). Prediction of enjoyment in school physical education. Journal of sports science \& medicine, $11(2), 260$.

Harness G (2001). Organizing participation in crosssex jump rope: Situating gender differences within longitudinal studies of activities. Research on Language \& Social Interaction 34(1): 75-106. https:/ / doi. org/ 10.1207/ S15327973RLSI3401_4

Harter, S. (1999). The construction of the self. NuevaYork: Guilford.

Jang, H., Reeve, J., \& Deci, E. L. (2010). Engaging students in learning activities: It is not autonomy support or structure but autonomy support and structure. Journal of Educational Psychology, 102, 588600. doi:10.1037/ 20019682

Julián, J. A., A barca-Sos, A., Aibar, A., Peiró-Velert, C., \& Generelo, E. (2010). La observación sistemá tica como instrumento de análisis del clima motivacional en Educación Física. M otricidad. European Journal of H uman M ovement, 25, 119-142.

Lee, A. M. (2002). 2001 CH M cCloy research lecture: Promoting quality school physical education: Exploring the root of the problem. Research Q uarterly for Exercise and Sport, 73(2), 118-124. https:/ / doi.org/ 10.1080/ 02701367.2002.10609000

Lobo,Y. B. , \&W insler, A. (2006). The effects of a creative dance and movement program on the social competence of head start preschoolers. Social Development, 15(3), 501-519. https:/ / doi.org/ 10.1111/ j.1467-9507.2006.00353.x

López, J. C. , \& Sánchez, G. S. (2010). Educación artísticapor el movimiento: la expresión corporal en edu- 
cación física. Aula, 16, 113-134.

López, J. C., \& Sánchez, G. S. (2012). Expresión corporal en educación física: la construcción de una disciplina. EmásF: revista digital deeducación física, (14), 164-175.

López, J. C., \& Sánchez, G. S. (2013). Expresión Corporal en Educación Física: bases para una didáctica fundamentada en los procesos creativos. Retos. Nuevas tendencias en educación física, deporte y recreación, (24), 117-122.

Martín, M. R. R. (2015). Expresión corporal en educación física (Vol. 245). Prensas de la U niversidad de Zara goza.

Martínez, C., Alonso, N., \& Moreno, J. A. (2006). Aná lisis factorial confirmatorio del «Cuestionario de Percepción de Éxito (POSQ)» en alumnos adolescentes de Educación Física. En M. A. González, J. A. Sánchez \& A. Areces (Eds.), IV Congreso de la Asociación Española de Ciencias del Deporte (pp. 757-761). A Coruña: Xunta de Galicia.

Maset, P. P. (2012). Aulas inclusivas y aprendizaje cooperativo. Educatio Siglo XXI, 30(1), 89-112.

Mitchell, F., Gray, S., \& Inchley, J. (2015). 'This choice thing really works... 'Changes in experiences and engagement of adolescent girls in physical education classes, during a school-based physical activity programme. Physical Education and Sport Pedagogy, 20(6), 593-611. https:// doi.org/ 10.1080/ 17408989.2013.837433

Montávez, M. (2003). Los valores humanos a través de la Expresión Corporal. In Expresión, Creatividad y M ovimiento. Actas del I Congreso Internacional de Expresión Corporal y Educación.

Moreno-Murcia, J. A., Gimeno, E. C., Carretero, C. M., Lacárcel, J. A. V., \& Calvo, T. G. (2012). M etas sociales, necesidades psicológicas básicas y motivación intrínseca como predictores de la percepción del esfuerzo en las clases de educación física. Revista depsicología del deporte, 21(2), 215-221.

Moreno-Murcia, J. A., \& Sánchez-Latorre, F. (2016). The effects of autonomy support in physical education classes. Revista Internacional de Ciencias del Deporte, 12 (43), 79-89. http:/ / dx.doi.org/ 10.5232/ ricyde2016.04305

Moreno-M urcia, J. A., Gonzalez-Cutre, D., Chillon, M. $\&$, Parra, N. (2008). Adaptación a la educación física de la escal a de las necesidades psicológicas básicas en el ejercicio. Revista M exicana de Psicología, 25(2), 295303.

Morgan, K., \& Kingston, K. (2008). Development of a self-observation mastery intervention programme for teacher education. Physical Education and Sport Pedagogy, 13(2), 109-129. https:/ / doi.org/ 10.1080/ 17408980701345634

Morgan, K., Sproule, J., Weigand, D. \& C Carpenter, P. (2005). A computer-based observational assessment of the teaching behavioursthat influence motivational climate in physical education. Physical Education \& Sport Pedagogy, 10(1), 83-105. https:/ / doi.org/ $10.1080 / 1740898042000334926$

Mosston, M. (1978). La enseñanza de la Educación Física. Del comando al descubrimiento. Paidós. Barcelona.

Murillo, B. , Julián, J. A. , García-González, L., Abarca-

Sos, A. \& Zaragoza, J. (2014). Influencia del género y de los contenidos sobre la actividad física y la percepción de competenciaen Educación Física. RICYDE. Revista Internacional deCienciasdel D eporte, 10 (36), 131143. http:/ / dx. doi.org/ 10.5232/ ricyde2014.03604 Newton, M., Duda, J. L., \&Yin, Z. (2000). Examination of the psychometric properties of the Perceived Motivational Climate in Sport Q uestionnaire-2 in a sample of female athletes. Journal of Sports Sciences, 18(4), 275-290. https:/ / doi.org/ 10.1080/ 026404100365018

Nicholls J (1989). The Competitive Ethos and Democratic Education. Cambridge, MS: Harvard U niversity Press.

0 'N eill, J. R., Pate, R. R., \& Liese, A. D. (2011). Descriptive epidemiology of dance participation in adolescents. Research quarterly for exercise and sport, 82(3), 373-380. https:// doi.org/ 10.1080/ 02701367.2011.10599769

Ordás, R. P., Lluch, Á. C., \& Sánchez, I. G. (2012). U na metodología para la expresión corporal actual en el ámbito educativo y recreativo. EmásF: revista digital de educación física, (14), 39-51.

Pérez, L. M. R., Sanz, J. L. G., Sánchez, I. R., \& Mata, E. (2004). Preferencias participativas en educación física de los chicos y chicas de la educación secunda ria mediante la «la escala de participación social en el aprendizaje. European Journal of Human Movement, 12, 157-174.

Reeve, J. (2006). Teachersasfacilitators:W hat autonomysupportive teachers do and why their students benefit. The Elementary School Journal, 106, 225-236. doi: 10.1086/ 501484

Riemer, B. A., \& Visio, M. E. (2003). Gender typing of sports: An investigation of Metheny's classification. Research Quarterly for Exercise and Sport, 74 (2), 193-204. https:/ / doi.org/ 10.1080/ 
02701367.2003 .10609081

Roberts, G. C., \& Balagué, G. (1991).The development and validation of the Perception of Success Q uestionnaire. In FEPSAC Congress, Cologne, Germany.

Roberts, G. C., Treasure, D. C., \& Balague, G. (1998). Achievement goals in sport: The development and validation of the Perception of Success Q uestionnaire. Journal of Sports Sciences, 16(4), 337347. https:/ / doi.org/ 10.1080/ 02640419808559362

Rodríguez, J. R. (2002). Trabajo de campo sobre el análisis del juego infantil en Primaria. In Vamos a jugar: El juego de primaria (pp. 151-163). Universidad de Huelva.

Ruano-Arriagada, M.T., y Barriopedro, M.I. (2006). Impacto de la expresion corporal sobre las emocionesen el ambito educativo. European Journal of $\mathrm{H}$ uman M ovement, (15), 1-7.

Rueda, B. (2004). La expresion corporal en el desarroIlo del area de educacion fisica. En Castillo, E.; Diaz, M. (2004). Expresion Corporal en Primaria. Servicio de publicaciones. Universidad de Huelva.

Ruiz, L. M., Graupera, J. L., M oreno, J. A., \& Rico, I. (2010). Social preferences for learning among adolescents in secondary physical education. Journal of Teaching in Physical Education, 29(1), 3-20. https:/ / doi.org/ 10.1123/ jtpe.29.1.3

Ryan, R. M., \& Deci, E. L. (2000). Self-determination theory and the facilitation of intrinsic motivation, social development, and well-being. American psychologist, 55(1), 68.

Ryan, R. M., Soenens, B., \& Vansteenkiste, M. (2019). Reflections on self determination theory as an organizing framework for personality psychology: Interfaces, integrations, issues, and unfinished business. Journal of personality, 87 (1), 115-145. https: / / doi.org/ 10.1111/ jopy. 12440

Sánchez, I. G., Ordás, R. P., \& Lluch, Á. C. (2011). Iniciación a la danza como agente educativo de la expresión corporal en la educación física actual: aspectos metodológicos. Retos: nuevas tendencias en educación física, deporte y recreación, (20), 33-36.

Sánchez-O liva, D., Viladrich, C., Amado, D., GonzálezPonce, I., \& García-Calvo, T. (2014). Predicción de los Comportamientos Positivos en Educación Física: una perspectiva desde la Teoría de la Autodeterminación. Revista de psicodidáctica, 19(2), 387-406. DOI: 10.1387/ RevPsicodidact. 7911

Sampol, P. P., Verdaguer, X. P., Planas, M. G., Rotger, P. A. B., \& Conti, J. V. (2005). Motivos para el inicio, mantenimiento y abandono de la práctica deportiva de los preadolescentes de la isla de Mallorca. Apunts Educación Física y Deportes, (81), 5-11.

Schinca, M. (2000). Expresion corporal: Tecnica y expresion del movimiento. Barcelona: Praxis

Sevil, J., Abós, Á., Aibar, A., Julián, J. A. y GarcíaGonzález, L. (2016). Gender and corporal expression activity in physical education: Effect of an intervention on students' motivational processes. European Physical Education Review, 22(3), 372-389. https: / / doi.org/ $10.1177 / 1356336 \times 15613463$

Tessier, D., Sarrazin, P., \& N toumanis, N. (2010). The effect of an intervention to improve newly qualified teachers' interpersonal style, student's motivation and psychological need satisfaction in sport-based physical education. Contemporary Educational Psychology, 35(4), 242-253. https:/ / doi.org/ 10.1016/ j.cedpsych.2010.05.005

Vallés-Arándiga, A., \& VallésTortosa, C. (2000). Inteligencia emocional: aplicaciones educativas (No. Sirsi) i9788489967816)

Vansteenkiste, M., \& Ryan, R. M. (2013). On psychological growth and vulnerability: basic psychological need satisfaction and need frustration as a unifying principle. Journal of psychotherapy integration, 23(3), 263. https:/ / doi.org/ 10.1037/ 20032359

W hitehead, S., \& Biddle, S. (2008). Adolescent girls' perceptions of physical activity: A focus group study. European physical education review, 14(2), 243262. https:/ / doi.org/ 10.1177/ 1356336X 08090708 Vlachopoulos, S. P., \& Michailidou, S. (2006). Development and initial validation of a measure of autonomy, competence, and relatedness in exercise: The Basic Psychological Needs in Exercise Scale. Measurement in physical education and exercise science, 10(3), 179-201. https:/ / doi.org/ 10.1207/ s15327841mpee1003 4

W illiams, G. C., Saizow, R., Ross, L., \& Deci, E. L. (1997). M otivation underlying career choice for internal medicine and surgery. Social science $\&$ medicine, 45(11), 1705-1713. https:/ / doi. org/ 10.1016/ S0277-9536(97)00103-2.

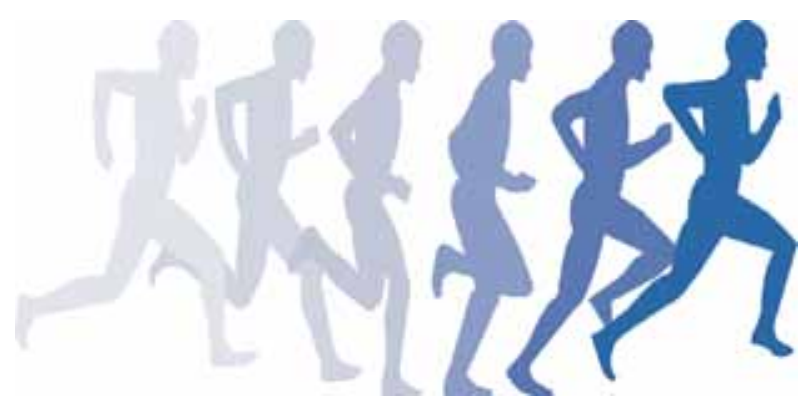

